\title{
Attackability Characterization of Adversarial Evasion Attack on Discrete Data
}

\author{
Yutong Wang* \\ King Abdullah University of Science \\ and Technology \\ Thuwal, Saudi Arabia \\ 11611808@mail.sustech.edu.cn \\ Yun Shen \\ Nortonlifelock Research Group \\ Reading, United Kingdom \\ yun.shen@nortonlifelock.com
}

\author{
Yufei Han \\ Nortonlifelock Research Group \\ Sophia-Antipolis, France \\ yufei.han@nortonlifelock.com
}

\author{
Fenglong Ma \\ Penn State University \\ State College, Pennsylvania, USA \\ fenglong@psu.edu \\ Xiangliang Zhang ${ }^{\dagger}$ \\ King Abdullah University of Science \\ and Technology \\ Thuwal, Saudi Arabia \\ xiangliang.zhang@kaust.edu.sa
}

\author{
Hongyan Bao \\ King Abdullah University of Science \\ and Technology \\ Thuwal, Saudi Arabia \\ hongyan.bao@kaust.edu.sa \\ Jin Li \\ Guangzhou University \\ Guangzhou, China \\ jinli71@gmail.com
}

\begin{abstract}
Evasion attack on discrete data is a challenging, while practically interesting research topic. It is intrinsically an NP-hard combinatorial optimization problem. Characterizing the conditions guaranteeing the solvability of an evasion attack task thus becomes the key to understand the adversarial threat. Our study is inspired by the weak submodularity theory. We characterize the attackability of a targeted classifier on discrete data in evasion attack by bridging the attackability measurement and the regularity of the targeted classifier. Based on our attackability analysis, we propose a computationally efficient orthogonal matching pursuit-guided attack method for evasion attack on discrete data. It provides provably computational efficiency and attack performances. Substantial experimental results on real-world datasets validate the proposed attackability conditions and the effectiveness of the proposed attack method.
\end{abstract}

\section{CCS CONCEPTS}

- Theory of computation $\rightarrow$ Adversarial learning; • Computing methodologies $\rightarrow$ Optimization algorithms; Discrete space search; Neural networks.

\section{KEYWORDS}

Attackability, Evasion Attack, Discrete Data, LSTM, Deep Learning

\section{ACM Reference Format:}

Yutong Wang, Yufei Han, Hongyan Bao, Yun Shen, Fenglong Ma, Jin Li, and Xiangliang Zhang. 2020. Attackability Characterization of Adversarial Evasion Attack on Discrete Data. In Proceedings of the 26th ACM SIGKDD Conference on Knowledge Discovery and Data Mining (KDD '20), August 23-27, 2020, Virtual Event, CA, USA. ACM, New York, NY, USA, 11 pages. https://doi.org/10.1145/3394486.3403194

\footnotetext{
*Intern student at King Abdullah University of Science and Technology.

${ }^{\dagger}$ Corresponding author
}

\section{INTRODUCTION}

Despite fruitful progress of evasion attack on continuous data, such as images and videos $[2-4,7,8,8,18,20,31,32]$, how to design adversarial examples for discrete data remains a rarely investigated, but important research problem. Discrete data pervasively exists in real-world applications and analytic practitioners have been designed for financial fraud detection, spam email detection, gene analysis in bioinformatics and malware dynamic analysis, etc. Many of these applications are safety-critical, while eager to deploy machine learning technologies. Investigating the adversarial vulnerability on discrete data thus becomes a must in these industrial practices.

Real-world discrete data are usually located in a space spanned by physically meaningful dimensions. For example, the presence or absence of a keyword in a document, a gene in a genome or a function call of an executable file. The discrete attributes of an object form a highly structured and semantic description of the object. Compared to the low-level and continuous measurements like pixel intensities, discrete attributes and their combination encode highlevel qualitative concepts. Pioneering work of this topic, such as evasion attack against spam filtering and NLP classifiers $[14,16$, $17,21,22,27,30,33,34]$, depends heavily on the domain-specific knowledge about feature extraction. In general, adversarial evasion attacks on discrete data raise the following fundamental challenges.

First of all, designing adversarial modifications of discrete data is intrinsically an NP-hard combinatorial optimization task. Similar to the problem in continuous domain, the solvability of an evasion attack task on discrete data (a.k.a. attackability) is closely related to the characteristics of the attack objective and the targeted classifier. The key questions about characterizing the attackability are thus: 1) What conditions does the attack objective need to meet to define a tractable attack problem? 2) Can we provide provably attack performances? 
Both questions are open according to the current research progress. Given the discontinuity nature of discrete data, modifying any of the attributes might cause a big leap in the discrete feature space. Gradient-based attack methods, though pervasively used for continuous data, cannot be applied directly. Classically, combinatorial optimization problems are [26] relaxed to continuous linear or quadratic programming tasks. However, such solutions require a simple form of the targeted classifier, like linear models. They become infeasible when a highly non-linear deep neural network based classifier is used. Recent works on graph poisoning attack $[1,10,12]$ and evasion attack against NLP classifiers [29] use greedy search to solve the attack problem. Notably, these works formulate evasion attack on discrete data as a problem of submodular function maximization. Benefited from submodularity of the attack objective, the greedy search based attack methods in these works enjoy strong performance guarantees. However, it is still unclear how to evaluate the attackability of a general evasion attack. Furthermore, enforcing strict submodularity limits the choice of the targeted classifier and/or harm the usability of the targeted classifier by introducing artifacts into the classifier.

Secondly, it is difficult to find out the physical meaning of the adversarial noise for continuous data. Especially for images, the intensity change of individual pixels is not necessarily associated with any meaningful characteristics of the visual contents. In contrast, a change of discrete attributes indicates a drift in the concept space spanned by the discrete attributes and implies the sensitivity of each attribute for the classification task. Therefore, beyond delivering a successful evasion from the targeted classifier, we expect to reveal the answer to the question, "Can we associate the adversarial modification of the discrete attributes with any characteristics of the classification task, e.g. sensitivity of the attributes?"

We echo the challenges by providing attackability guarantees of white-box evasion attack against non-linear classifiers, such as deep neural nets, on discrete data. We aim at flipping the classification output while preserving the integrity of the data as much as possible. Notably, we save for the future to address the issue of maintaining the functionality of data after the attack, e.g., preserving applicability of malwares or readability of texts. On one hand, defining the rules to preserve the data functionality depends on domain-specific knowledge. On the other hand, it doesn't change the combinatorial nature of the attack problem. Our attackability study can still be applied by integrating a-priori data functionality-preserving rules. We summarize our contributions as follows:

- We cast the evasion attack on discrete data as a set function optimization problem. We show that the attack problem is solvable if the targeted classifier follows certain regularity constraints. Benefited from these constraints, the objective function of the attack problem enjoys weak submodularity, which makes the generally NP-hard problem feasible to be solved approximately with polynomial complexity by greedy search based methods. We instantiate the attackability study on recurrent neural network (RNN) with discrete data.

- We reveal that the solution quality of the greedy search based attack depends on the regularity of the targeted classifier, which determines the submodularity ratio of the attack objectives. It defines a balance between the modeling flexibility and attackability of the targeted classifier. Fewer regularity constraints enable broader choices of the classifiers' architectures. However, it makes the attack problem more difficult to solve simultaneously. Furthermore, to address the computational bottleneck of the primitive greedy search, we propose an orthogonal matching pursuit based greedy search to improve the efficiency of the solution. The proposed method provides a bounded estimator of the marginal gain of the attack objective with respect to each attribute. It only selects and perturbs the attributes of the largest marginal gain, fastening the attack while guaranteeing its quality.

- In addition, we explore the association between the solution to the attack and sensitivity measurement of the attributes. We extend the evasion attack to flag commonly useful attributes to attack different instances. Despite a lack of theoretical explanation, we observe an interesting consistency between the most sensitive attributes and the most commonly selected attributes to attack.

\section{RELATED WORK}

Most research efforts of evasion attack with discrete input focus on attacking NLP classifiers by modifying individual words or sentences. Such attack methods use heuristic rules, e.g. replacing words with candidate synonyms defined manually [14, 16, 21, 22, 27, 30, 33]. These works borrow directly the key idea of the evasion attack in continuous domain. They choose the discrete word/sentence transformations, which are mostly aligned with the gradient vector of the attack objective. Similarly [17] proposes to conduct Carlini's gradient-guided evasion attack [7] on continuous word-embedding space first. It generates a target embedding representation of the text. The method then searches for the candidate entities carrying the most similar embedding vector with the targeted embedding representation to replace the corresponding words/sentences in the original text. These methods don't provide provable guarantees of a successful attack. On one hand, the word embedding space is non-smooth. The embedding vectors of semantic synonyms can be so different that they don't appear as neighbors in the embedding space. On the other hand, nevertheless, the gradient vector of the attack objective only approximates local variations around the input embeddings. Modifying even one word/phrase can violate this assumption in the embedding space.

Submodular function optimization has been introduced as a decent solution to the NP-hard combinatorial optimization since 1970s [23-25]. For monotone submodular function maximization, primitive greedy search with polynomial complexity can achieve an approximation ratio of $1-1 / e$. In evasion attack on discrete data, e.g., edge-flipping based attack against graph embeddings, the use of submodular functions has been witnessed in [1, 10, 12, 29]. Though it is not explicitly claimed, $[1,10,12]$ define the attack objective as a sum of smallest eigenvalues of the adjacency matrix of a given graph. It is intrinsically a problem of submodular function maximization. [29] introduces additional positiveness constraint over the $\mathrm{CNN}$ and RNN based classifier's parameters. The resultant classifiers are proven to be submodular. A greedy search based attack method is then used to select the targeted words/sentences and replace them with feasible candidates. 
Despite of the effectiveness, the use of submodular function is limited, especially in adversarial learning research. The objective functions adopted in practices usually deviate from strict submodularity. In this case, greedy search can perform arbitrarily poorly in general. Besides, enforcing the submodularity constraint on the targeted classifiers introduces artefacts into the classifiers' architectures and limits the model choice. It can cause degradation of classification utility. Therefore, such attack model is still far from the adversarial threat of real-world practices. Recent research efforts break the hurdle by introducing $\gamma$-weakly submodular functions $[9,11]$. Submodularity ratio $\gamma$ measures the distance of the function from being strictly submodular. The standard greedy algorithm achieves a graceful approximation ratio of $1-e^{-\gamma}$ for the problem of maximizing such functions subject to a cardinality constraint.

It is a brand new problem to shape evasion attack on discrete data with weak submodularity theory. Intuitively, weak submodularity can broaden the choice of the targeted classifier for feasible attacks, which helps to define more realistic adversarial threat scenarios. Nevertheless, it is unclear how to design a weakly submodular objective function for the attack. The celebrated work by [13] sets up an equivalence between the smoothness and strongly concavity of log-likelihood objective functions and its weak submodularity in a feature subset selection problem. Inspired by this work, we reveal that the evasion attack can be formulated as a problem of weakly submodular function maximization, if the targeted classifier follows even less strict regularity constraints. We argue that enforcing strong concavity over the classifier is not obliged to guarantee weak submodularity. We further characterize the attackability of evasion attack given the regularity of the targeted classifier.

\section{ATTACKABILITY ANALYSIS}

We use $\mathbf{x}=\left\{x_{1}, x_{2}, x_{3}, \ldots, x_{n}\right\}$ to represent an instance with $n$ discrete features. Each $x_{i}$ is a cell of $m(m \geq 1)$ categorical attributes. For example, an $x_{i}$ can be a code segment in a malware file associated with one unique function or a medical examination output linked to the $m$ different biological characteristics of human body. In practice, we cast each categorical attribute value to a $D$-dimensional pre-trained embedding vector, e.g., $\mathrm{e}^{j} \in R^{D}, j=1,2, \ldots, m$.

To represent instance $\mathbf{x}$ by the embedding vectors of its attribute values, we introduce binary variables $\mathbf{b}=\left\{b_{i}^{j}\right\}, i=1,2, \ldots, n$, $j=1,2, \ldots, m$, where $b_{i}^{j}=1$ when the $j$-th attribute value is selected for $x_{i}$, and $b_{i}^{j}=0$ otherwise. One instance $\mathbf{x}$ encoded by the embedding vectors can then be represented as an $R^{n * m * D}$ tensor with $\mathbf{x}_{\{i, j,:\}}=b_{i}^{j} e_{i}^{j}$.

We use $\hat{\mathbf{b}}=\left\{\hat{b}_{i}^{j}\right\}$ as the adversarially tuned variable modified from $\mathbf{b}$. If no modification, $\hat{b}_{i}^{j}=b_{i}^{j}$. Otherwise, $\hat{b}_{i}^{j} \neq b_{i}^{j}$. Depending on the type of attacks to implement, e.g., insertion, deletion or substitution, $\hat{b}_{i}^{j}$ can have different values. Insertion is to let $\hat{b}_{i}^{j}=1$, given $b_{i}^{j}=0, \forall j=1, \ldots, m$. Deletion is to let $\hat{b}_{i}^{j}=0$, given $b_{i}^{j}=1$. Substitution is to let $\hat{b}_{i}^{j}=1, \hat{b}_{i}^{j^{\prime}}=0$, given $b_{i}^{j}=0, b_{i}^{j^{\prime}}=1, j \neq j^{\prime}$. A modified instance $\hat{\mathbf{x}}$ can thus be written as $\hat{\mathbf{x}}_{\{i, j,:\}}=\hat{b}_{i}^{j} e_{i}^{j}$.

Let $\mathbf{y}$ be the target class label of the evasion attack. The goal is to make $f_{y}(\hat{\mathbf{x}})$ deviate from $f_{y}(\mathbf{x})=0$. In other words, we aim at maximizing $f_{y}(\hat{\mathbf{x}})$, so that $\mathbf{x}$ is modified to get $\mathbf{y}$ assigned to it.
The evasion attack task can then be formulated as a process of set function optimization, defined as

$$
\begin{aligned}
& \qquad S^{*}=\underset{|S| \leq K}{\arg \max } g(S) \\
& \text { where } \quad g(S)=\max _{l \subset S} f_{y}(\hat{\mathbf{x}}), \quad l=\operatorname{diff}(\mathbf{b}, \hat{\mathbf{b}})
\end{aligned}
$$

where $|S|$ is the cardinality of set $S$. Function $g(S)$ is a set function measuring the maximum extent to which the attacks in $S$ can change the classification result. The diff function reports the set of the indices where $\mathbf{b}$ and $\hat{\mathbf{b}}$ are different. In other words, $l$ denotes the set of modification to make for attacking $\mathbf{x}$. To preserve data integrity, the modification made on $\hat{\mathbf{b}}$ should be as small as possible $(|S| \leq K)$.

Obviously $g(S)$ is a non-decreasing monotone set function as $g(S) \leq g(T)$ if $S \subseteq T$. By solving Eq. (1), we pursue to find the optimal set of adversarial discrete attribute modification, including adding, deleting and replacing values of discrete attributes in the original data instance.

\subsection{Weak Submodularity Based Solvability Conditions}

To solve the attack problem in Eq.(1), we first evaluate its solvability conditions based on weak submodularity [11]: we require the attack objective to be weakly submodular and show it can be solved approximately with polynomial complexity given this constraint. We introduce submodularity ratio [11] to measure the attackability of the evasion attack problem defined by Eq.(1). For a weakly-submodular attack objective, the higher the submodularity ratio is, the better attack solution we can obtain via maximizing the attack objective, while the less constraints we need to enforce over the classification model.

We elaborate our solution by first introducing the definition of weak submodularity and submodularity ratio.

Definition 1. Given a set cardinality threshold $k \geq 1$, the submodularity ratio $\gamma_{k}$ of a set function $g($.$) w.r.t. a set H$ is:,

$$
\gamma_{k}=\min _{S \subseteq H, A:|A| \leq k, S \cap A=\emptyset} \frac{\sum_{a \in A} g(S \cup a)-g(S)}{g(S \cup A)-g(S)}
$$

If $\gamma=\min _{k} \gamma_{k}<1$, the set function $g(S)$ is weakly submodular. Otherwise, $g(S)$ is submodular when $\gamma \geq 1$ for any $S$.

We define the regularization constraint over the classification function $f_{y}$ by extending Restricted Strong Convexity (RSC) in Theorem.1 of [13] to apply to non-concave functions. It guarantees further weak submodularity of the attack objective of broader classes of function forms.

Definition 2. Let $\Omega=(x, y), x, y \in R^{n}$ and $\ell: R^{n} \rightarrow R$ be a continuously differentiable function. A function $f$ is $\left(m_{\Omega}, M_{\Omega}\right)$-bounded on $\Omega$, iffor any $(x, y) \in \Omega, m_{\Omega} \in R$ and $M_{\Omega} \in R^{+}$:

$$
\begin{aligned}
& f(y)-f(x)-\langle\nabla f(x), y-x\rangle \geq-\frac{M_{\Omega}}{2}\|y-x\|_{2}^{2} \\
& f(y)-f(x)-\langle\nabla f(x), y-x\rangle \leq-\frac{m_{\Omega}}{2}\|y-x\|_{2}^{2}
\end{aligned}
$$

REMARK 1. If $m_{\Omega}>0, f$ is then strongly concave. If $m_{\Omega} \leq$ $0, f$ may violate the concavity constraint and presents linearity or convexity. It is easy to find that, larger $M_{\Omega}$ and smaller $m_{\Omega}$ relax the bounded constraint enforced to the function $f$, which allows $f$ to choose among a broader class of functions. 
Input: The attack budget $K$, the set function $g(S)$ defined by Eq. (1) and the set $H=\{(i, j), i=1 \ldots n, j=1 \ldots m\}$ of all the modifiable discrete values

Output: selected support set $S_{k}$, with $\left|S_{k}\right| \leq K ; g\left(S_{k}\right)$ and the optimal subset of $S_{k}$ achieving the attack goal

$S_{0} \leftarrow \emptyset$

for $k=1$ to $K$ do

$s \leftarrow \arg \max g\left(S_{k-1} \cup j\right)-g\left(S_{k-1}\right)$

$j \in H / S_{k-1}$

$S_{k} \leftarrow S_{k-1} \cup\{s\}$

end

Algorithm 1: Forward Stepwise Greedy Search (FSGS)

We then present the regularity constraint enforced on $f_{y}$ to guarantee weak submodularity of the attack objective in Theorem 1. We then link the lower bound of the submodularity ratio (how "weakly submodular" the attack objective is) to the approximation quality of Eq. (1) in Theorem 2. Both theorems form the base of the attackability study of the evasion attack on discrete data.

THEOREM 1. Let $b$ as the unchanged original binary indicator defined in Eq.1. Let $\Omega_{k}=\left\{\left(\hat{b}, \hat{b}^{\prime}\right):|\operatorname{diff}(b, \hat{b})| \leq k,\left|\operatorname{diff}\left(b, \hat{b}^{\prime}\right)\right| \leq\right.$ $k$, $\left.\left|\operatorname{diff}\left(\hat{b}, \hat{b}^{\prime}\right)\right| \leq k\right\}$, where $\hat{b}$ and $\hat{b}^{\prime}$ denote two sets of selected discrete attributes to be modified adversarially. If the classifier $f_{y}$ is $\left(m_{\Omega_{k}}, M_{\Omega_{k}}\right)$-regularized on $\Omega_{k}$, the $g(S)$ defined by Eq.1 is weakly submodular. Its submodularity ratio $\gamma_{k}$ on $\Omega_{k}$ is bounded from below:

$$
\begin{aligned}
\gamma_{k} & \geq \frac{1}{2 \psi_{k} M_{\Omega_{k}}} \\
\psi_{k} & =1+\frac{k^{2}\left|m_{\Omega_{k}}\right|}{2\left\|\nabla f_{y}(b)_{s}\right\|_{2}^{2}}, \text { If } m_{\Omega_{k}} \leq 0 \\
\psi_{k} & =\frac{1}{2 m_{\Omega_{k}}}, \text { If } m_{\Omega_{k}}>0
\end{aligned}
$$

where $\nabla f_{y}(b)_{v}$ denotes the elements of $\nabla f_{y}(b)$ corresponding to the difference between the index sets $l_{b}$ and $l_{b^{\prime}}$, where $v=l_{b^{\prime}} \backslash l_{b}+l_{b} \backslash l_{b^{\prime}}$.

According to Theorem.1, if the targeted classifier $f_{y}$ has a well regularized function form as required by Eq.3, the objective function of the evasion attack defined by Eq.1 then enjoys weak submodularity with a bounded submodularity ratio. As proved in [11], such a weakly submodular attack objectivecan be maximized by incrementally changing discrete attributes. This primitive greedy search based solution is also known as Forward Stepwise Greedy Search (FSGS) [13]. More specifically, let $S$ denote the set of modified attributes in the previous iterations. In next iteration, FSGS finds $s=(i, j)$ to add to $S$, if $\hat{b}_{i}^{j}$ is set to be different from $b_{i}^{j}$ to achieve the largest marginal gain of $g(S \cup s)$ over $g(S)$. The algorithm is represented in Algorithm.1.

This primitive greedy search method is computationally expensive, because in each iteration, FSGS evaluates all the unchanged values to select the next candidate to modify. That's to say, $s$ is selected from all the unchanged values to see which makes maximum improvement of $g(S \cup s)$ over $g(S)$. In each iteration, FSGS needs $O\left(\left(\sum_{i=1}^{k-1} C_{k}^{i}\right)(|H|-\kappa)\right)$ objective function evaluations to search the optimal candidate attribute $s$, where $|H|$ denotes the size of all feasible candidate attributes to change and $\kappa=\left|S_{k}\right|$. As seen, the computational cost of FSGS becomes quickly expensive with increasingly larger $\left|S_{k}\right|$. It is the major bottleneck of FSGS. We discuss how to address this issue and deliver faster while successful attack later in Section.4.

THEOREM 2. [Theorem 3 in [13]] Let the evasion attack problem defined by Eq.(1) be with the classification function $f_{y}$ that is $\left(m_{\Omega_{k}}, M_{\Omega_{k}}\right)$-bounded. Let $S_{k}$ be the set of the values selected by FSGS and $S_{k}^{*}$ be the underlying optimal value set following the support size constraint. The corresponding attack objective values reached by $S_{k}$ and $S_{k}^{*}$ are $g^{F S G S}$ and $g^{O P T}$, respectively. Then $g^{F S G S}$ is bounded:

$$
g^{F S G S} \geq\left(1-e^{-\gamma S_{k}}\right) g^{O P T}
$$

where $\gamma_{S_{k}}$ is the submodularity ratio of $g(S)$ defined on the selected set $S_{k}$. Especially, if $g(S)$ is submodular, the lower bound gives as:

$$
g^{F S G S} \geq\left(1-e^{-1}\right) g^{O P T}
$$

We can find explicitly the relation between the regularity of the classification $f_{y}$ and solvability of the attack objective as follows:

- Submodularity Ratio v.s. Regularity of $f_{y}$ : According to Theorem 1, a classification function $f_{y}$ following the bound conditions is guaranteed to be weakly submodular. A smoother function $f_{y}$ (smaller $M_{\Omega}$ ) with higher $m_{\Omega}$ (closer to concavity) can give a higher bound of the submodularity ratio of $g(S)$. It thus makes Eq. (1) closer to submodularity. In contrary, for a less regularized $f_{y}$, especially when $m_{\Omega_{k}} \leq 0$, the submodularity ratio of the attack objective deteriorates significantly.

- Solution quality v.s. Regularity of $f_{y}$ : According to Theorem 2 , the higher submodularity ratio the attack objective has, the better approximation quality the solution derived reaches, compared to the underlyingly optimal solution to the attack problem. In summary, with a more regularized function $f_{y}$, it is more likely to solve the NP-hard attack problem approximately with greedy search based solutions.

\subsection{Attackability of RNN}

We instantiate the attackability condition proposed in Theorem 1 to the case where recurrent neural network (RNN) based classifiers are applied. It is worth noting that the attackability analysis is not limited to RNN. We use this case study to demonstrate how to characterize the attackability for a given classifier on discrete data.

We consider a simple but generalizable RNN model $f_{y}(\mathbf{x})$ with $n$ time steps, taking an input of the $n$ discrete values in $\mathbf{x}$. The hidden state $h_{t}$ of RNN by taking $x_{t}$ is:

$$
h_{t}=\phi\left(\alpha^{T} h_{t-1}+\beta\left(\mathbf{b}_{t} \odot \mathbf{E}\right)+\theta\right)
$$

where $\alpha, \beta$ and $\theta$ are the parameters of the unit, and $\mathbf{b}_{t} \odot \mathbf{E}=$ $\left[b_{t}^{1} \mathbf{e}^{1} ; b_{t}^{2} \mathbf{e}^{2} ; \ldots ; b_{t}^{k} \mathbf{e}^{m}\right]$ is the representation of $x_{t}$ in its attribute value embedding form. The output of the classifier is given based on the hidden unit of the last time step, as $f_{y}(\mathbf{x})=\phi_{y}\left(w_{y}^{T} h_{n}+\theta_{y}\right)$, where $w_{y}$ and $\theta_{y}$ are the parameters of the classification layer, $\phi$ and $\phi_{y}$ are the activation function of each hidden unit and the classification layer. Notably, [5] defines deep submodular functions for text classification. Nevertheless, they are not applicable in the setting of adversarial attack.

According to Theorem 1, the attackability condition of evasion attack against the general form of RNN classifier can be given as: 
Corollary 2.1. Weakly Submodular Attack Objective. Given the classifier as $f_{y}(\mathbf{x})$ and the domain $\Omega_{k} \in R^{n} * R^{n}, \phi$ and $\phi_{y}$ can be chosen as Sigmoid and Tahn functions. The activation function of $f_{y}$ is thus non-concave and $m_{\Omega_{k}}$ of $f_{y}$ is negative. The corresponding attack objective function $g(S)$ defined by Eq. (1) is weakly submodular.

REMARK 2. Let $\rho$ be the set of values selected to attack and $|\rho|$ be the attack budget. The submodularity ratio $\gamma$ of $g(S)$ on $\Omega_{|\rho|}$ is bounded from below as:

$$
\begin{aligned}
& \gamma_{\Omega_{|\rho|}} \geq \frac{|\rho|^{2}\left|\nabla f_{y}(\mathbf{b})_{\rho}\right|}{4\left(2+\left|\nabla f_{y}(\mathbf{b})_{\rho}\right|\right)\left(2+\left|\nabla f_{y}(\mathbf{b})_{\rho}\right|+\left\|\nabla f_{y}(\mathbf{b})_{\rho}\right\|_{2}^{2}\right)} \\
& (\text { Tanh activation function) } \\
& \gamma_{\Omega_{|\rho|}} \geq \frac{|\rho|^{2}\left|\nabla f_{y}(\mathbf{b})_{\rho}\right|}{4\left(1+\left|\nabla f_{y}(\mathbf{b})_{\rho}\right|\right)\left(1+\left|\nabla f_{y}(\mathbf{b})_{\rho}\right|+\left\|\nabla f_{y}(\mathbf{b})_{\rho}\right\|_{2}^{2}\right)} \\
& \text { (Sigmoid activation function) }
\end{aligned}
$$

where $\left|\nabla y_{f}(\mathbf{b})_{\rho}\right|$ denotes the L1-norm of the gradient computed w.r.t. the original binary vector $\mathbf{b}$ without adversarial perturbation.

Enforcing additional constraints over the regularity of $f_{y}$ can make the attack objective function closer to submodularity. An example can be found as proposed in [29], where the parameters of the RNN-based classifier $f_{y}$ are forced to be positive to guarantee the hidden layer output to be positive. The resultant attack objective $g(S)$ is thus proved to be submodular. It can be considered as a special case of the attackability framework proposed by Theorem.1.

Corollary 2.2. Submodular Attack Objective. Let $f_{y}$ be the RNN classifier defined by Eq. (7). We assume that all the parameters of $f_{y}$ are positively valued. Let $\rho$ be the set of attributes selected to attack. Given the positiveness constraint, the activation function of $f_{y}$ is strictly concave and $m_{\Omega_{|\rho|}}$ of $f_{y}$ is positive. We can further derive $\frac{2\left(1+\left|\nabla f_{y}(\mathbf{b})_{\rho}\right|\right)}{|\rho|^{2}} \geq m_{\Omega_{|\rho|}}>0$ and $M_{\Omega_{|\rho|}} \geq \frac{2\left(1+\left|\nabla f_{y}(\mathbf{b})_{\rho}\right|\right)}{|\rho|^{2}}$. The submodularity ratio $\gamma_{\Omega_{|\rho|}} \geq 1$ of the corresponding $g(S)$. The attack objective is thus submodular.

Comparing Corollary 2.1 and Corollary 2.2, we can observe a balance between model usability and attackability. On one hand, though submodularity can improve the approximation quality of the greedy search based solution, enforcing the positiveness constraint inevitably introduces unnecessary bias into the classifier design. It can reduce the classification power of the classifier. On the other hand, as shown by Corollary.2.1, $f_{y}$ is free of the additional positiveness constraint. It loosens the bound condition by allowing $m_{\Omega}$ to be negative, which gives more freedom in choosing the form of $f_{y}$. However, the attack objective loses submodularity as the lower bound of the submodularity ratio degrades significantly.

\subsection{Link to Sensitivity Analysis of Attributes}

Though conducting sensitivity analysis of the attributes in a given classification task is beyond our scope, it is also interesting to observe that the evasion attack guided by weakly submodular function optimization prefers to modify sensitive attributes/features. Rather than only cheating the classifier, an adversarial attacker is more keen to gain simultaneously the task-specific knowledge of the defined features via the attack process. For example, the classifier's output might be highly sensitive to the perturbation over a specific subset of the discrete attributes. These attributes are the origin of adversarial vulnerability of the systems running in the given task. They can potentially be informative features for classification at the same time. On one side, unveiling the useful information can help the designer of the classifier to flag the attributes of physical significance. On the other side, it helps the adversarial attacker to understand the statistical characteristics of the training data for better attack and/or further training data information stealing. From the perspective of information security, such threat can be categorized as data privacy breach.

We can explore the risk induced by the evasion attack, that is, searching for the optimal support set of the attributes that can be used to successfully attack the classifier over multiple discrete data instances. We extend Eq.1 to apply it over a set of data instances:

$$
\begin{array}{ll} 
& S^{*}=\underset{|S| \leq K}{\arg \max } \sum_{r=1}^{R} g_{r}(S) \\
\text { where } \quad g_{r}(S)=\max _{l \subset S} f_{y}\left(\hat{\mathbf{x}}_{r}\right)
\end{array}
$$

where $g_{r}(S)$ is the attack objective function defined based on each discrete data instance $\mathbf{x}_{r}$. The notations of $g_{r}(S)$ follow those used in Eq. (1). As each $g_{r}(S)$ is at least weakly submodular, the sum of $g_{r}(S)$ is also weakly submodular. The submodularity ratio of the attack objective function over multiple data instances gives as $\min \left\{\gamma^{1}, \gamma^{2}, \ldots, \gamma^{R}\right\}$, where $\gamma^{r}$ is the submodularity ratio of each $g^{r}(S)$. We can still use greedy search to solve the attack problem and find out the set of discrete attributes that are generally useful for attacking multiple data instances. Intuitively, the output of the classifier $f_{y}$ should be statistically sensitive to adversarial perturbation over these identified attributes. In the empirical study, we confirm our intuition by first measuring attribute-wise sensitivity with the widely employed one-factor-at-a-time sensitivity analysis method [6]. We then observe the overlapping between the top-ranked attributes according to their sensitivity scores and the attributes selected and modified by the evasion attack in Section.5. A large intersection between these two sets of attributes indicates the high sensitivity of the selected attributes in the attack process. It implies the underlying association between the adversarial vulnerability of the targeted classifier and the feature sensitivity in the classification task.

\section{PROVABLE APPROXIMATION QUALITY OF GRADIENT-GUIDED GREEDY SEARCH}

The computational complexity of FSGS increases drastically as the number of the modifiable attributes in discrete instance $\mathbf{x}$ becomes larger. In our application datasets presented in next section, there can be more than $10 \mathrm{k}$ modifiable attribute values. This is the major bottleneck for applying FSGS practically. To address the issue, we propose an efficient attack solution based on orthogonal matching pursuit [28], which is referred as Orthogonal Matching Pursuit-based Greedy Search (OMPGS). This method narrows down the candidate attribute value modifications to those that correlate the best with the orthogonal complement of the attributes already selected to modify. These attribute values are identified by first calculating the gradient of $f_{y}(\mathbf{x})$ with respect to $\mathbf{b}$, denoted as $\nabla\left(f_{y}(\mathbf{b})\right)$. The candidate attributes to modify are chosen as the ones corresponding to the gradient elements with the largest magnitudes. We conduct 
greedy search only within these candidate features to choose the optimal attribute for adversarial tuning.

Algorithm 2 presents the pseudo codes of OMPGS. In each iteration, for each subset $l^{\prime}$ of the previous support set $S_{k-1}$, we compute the gradient of $f_{y}$ with respect to $\mathbf{b}_{l^{\prime}}$ (binary matrix $\mathbf{b}$ with the entries in $l^{\prime}$ changed). The top- $k^{\prime}$ attributes with the largest magnitudes in the gradient vector are selected to form a candidate set $s^{\prime}$. We can then find out the optimal attribute $s$ to extend for each subset $l^{\prime}$ and record the optimal attack objective value $g\left(S_{k-1} \cup s\right)$. In the outer iteration, we choose finally the attribute $j^{*}$ producing the largest marginal gain to add into $S_{k-1}$.

The worst case cost of objective function evaluation in each iteration of OMPGS is bounded by $O\left(\left(\sum_{i=1}^{k-1} C_{k}^{i}\right)\right)\left|k^{\prime}\right|$. We adjust $k^{\prime}$ to achieve a trade-off between enlarging the search range of greedy search and the cost of function evaluation. Usually $k^{\prime}$ is much less than $|H|-\left|S_{k}\right|$, especially when $H$ is significantly large (e.g. $H>10 \mathrm{k}$ ). Thus $O M P G S$ runs significantly faster than FSGS.

To further illustrate the rationality of the proposed $O M P G S$, we show that the magnitude of each element in $\nabla\left(f_{y}(\mathbf{b})\right)$ can be used to measure the marginal contribution of adding the corresponding attributes in the candidate set for adversarial attack.

Theorem 3. Gradient as Indicator. We assume $S$ and $T$ as two independent sets of the discrete attributes. $S \cap T=\emptyset .|T| \leq k$. Modification $\hat{\mathbf{b}}_{S \cup T}$ and $\hat{\mathbf{b}}_{S}$ are made by the optimal solution of the attack given the support set as $S \cup T$ and $S$, resulting in $\hat{\mathbf{x}}^{S \cup T}$ and $\hat{\mathbf{x}}^{S}$, respectively. Following the notations in Theorem 1, the lower bound of the marginal gain by adding extending the set $S$ to $S \cup T$ gives as:

$$
f_{y}\left(\hat{\mathbf{x}}^{S \cup T}\right)-f_{y}\left(\hat{\mathbf{x}}^{S}\right) \geq \frac{1}{2 M_{|S|+k}}\left\|\nabla f_{y}\left(\hat{\mathbf{b}}_{S}\right)_{T}\right\|_{2}^{2}
$$

where $M_{|S|+k}$ is the bound condition parameter defined on the domain $\Omega_{|S|+k}$ according to Eq.3. When $m_{|S|+k}>0$ in Eq.3, the attack objective $g(S)$ is submodular. The upper bound of the marginal gain can be further formulated as:

$$
f_{y}\left(\hat{\mathbf{x}}^{S \cup T}\right)-f_{y}\left(\hat{\mathbf{x}}^{S}\right) \leq \frac{1}{2 m_{|S|+k}}\left\|\nabla f_{y}\left(\hat{b}_{S}\right)_{T}\right\|_{2}^{2}
$$

If $m_{|s|+k} \leq 0$, the attack objective is weakly-submodular. The upper bound of the marginal gain gives as:

$$
f_{y}\left(\hat{\mathbf{x}}^{S \cup T}\right)-f_{y}\left(\hat{\mathbf{x}}^{S}\right) \leq \tilde{\psi}\left\|\nabla f_{y}\left(\hat{b}_{S}\right)_{T}\right\|_{2}^{2}
$$

where $\tilde{\psi}=\frac{\left|\nabla f_{y}\left(b_{S}\right)_{T}\right|}{\left\|\nabla f_{y}\left(b_{S}\right)_{T}\right\|_{2}^{2}}+\frac{m_{|S|+k} k^{2}}{2\left\|\nabla f_{y}\left(b_{S}\right)_{T}\right\|_{2}^{2}}$

According to Theorem 3, the magnitude of each element in the gradient vector derived in each iteration of Algorithm 2 provides a bounded estimate of the marginal gain by adding the corresponding attribute into the support set to attack. Intuitively, with the bounded estimate, we can shrink the candidate set for the attack in order to focus on the attributes that can potentially bring more improvement of the attack objective than the others. In this way, we reduce the number of attributes to traverse during the greedy search of each iteration, while preserving the solution quality as much as possible. Indeed, we can provide a provably lower bound of the approximation quality of $O M P G S$ as follows:

Theorem 4. With $\psi$ defined in Theorem 3 , let $S_{k}$ be the support set of the selected attributes to attack with OMPGS, the corresponding
Input: The attack budget $K$, the set function $g(S)$ defined by Eq. (1) and the set $H=\{(i, j), i=1 \ldots . . j=1 \ldots m\}$ of all the modifiable discrete attributes

Output: selected support set $S_{k}$, with $\left|S_{k}\right| \leq K ; g\left(S_{k}\right)$ and the optimal subset of $S_{k}$ achieving the attack goal

$S_{0} \leftarrow \emptyset$

for $k=1$ to $K$ do

$T=\emptyset$

for $l^{\prime} \subset S_{k-1}$ do

$r \leftarrow \nabla f_{y}\left(\mathbf{b}_{l^{\prime}}\right)$

$\mathbf{s}^{\prime}=\left\{s_{1}^{\prime}, s_{2}^{\prime}, \ldots, s_{k^{\prime}}^{\prime}\right\} \leftarrow \arg \max \left|<e_{j}, r>\right|$

$s \leftarrow \arg \max g\left(S_{k-1} \cup\{j\}\right)$

$j \in \mathbf{s}^{\prime}$

$T=T \cup\left\{\left(s, g\left(S_{k-1} \cup s\right)\right)\right\}$

end

$j^{*} \leftarrow \underset{j \in T}{\arg \max } g\left(S_{i-1} \cup\{j\}\right)$ $j \in T$

$S_{k} \leftarrow S_{k-1} \cup\left\{j^{*}\right\}$

end

Algorithm 2: Orthogonal Matching Pursuit based Greedy Search (OMPGS)

objective value $g^{O M P G S}$ is bounded from below as:

$$
\begin{aligned}
& g^{O M P G S} \geq\left(1-e^{m_{\Omega_{\mid}} S_{k}^{*}\left|M_{\Omega_{\mid}} S_{k}^{*}\right|}\right) g^{O P T},\left(m_{\Omega_{\mid} S_{k}^{*} \mid}>0\right) \\
& \left.g^{O M P G S} \geq\left(1-e^{1 /\left(2 \tilde{\psi} M_{\Omega_{\mid}} S_{k}^{*} \mid\right.}\right)\right) g^{O P T},\left(m_{\Omega_{\mid} S_{k}^{*} \mid} \leq 0\right)
\end{aligned}
$$

Theorem 4 gives the intuition that the performance of FSGS will be better than that produced by OMPGS, when the attack objective is submodular. FSGS and OMPGS have the same lower bound of the approximation quality. In addition, we find that the upper and lower bound of the marginal gain are tighter when the classifier $f_{y}$ is tightly bounded and the attack objective is submodular, compared to the weakly submodular opponent. If the classifier $y_{f}$ is well bounded to guarantee the submodularity, the gradient magnitude of $y_{f}$ provides a more accurate estimate of the marginal contribution of each attribute. OMPGS thus achieves a good balance between economic computing and effective greedy exploration.

\section{EXPERIMENTS}

\subsection{Dataset Information}

We include two real-world evaluation datasets, cyber security and electronic medical service, which are briefly introduced due to space limits. More information can be found in Appendix A.

Intrusion Prevention System Dataset (IPS) in Cyber Security. We collect one day of IPS records from 242,467 endpoint devices containing 29,641 intrusion events. Each intrusion instance is composed by 20 intrusion steps. On each intrusion step, one of 1,103 different malicious actions can be selected. We embed each action as a 70dim vector. Then one IPS data instance is given as $\mathbf{x} \in R^{20 * 1103 * 70}$ according to the definition given in Section 3. A classifier is built to predict if, given an intrusion, the next action would fall into 2 highly malicious actions and a third class for all others. The evasion attack is non-targeted. We aim at mis-classifying an $x$ to any of the two classes other than the true class label, by replacing the original action at a given intrusion step with a new action. 
Table 1: Attack Performances on IPS dataset

\begin{tabular}{|c|c|c|c|c|c|c|c|c|c|c|c|c|c|}
\hline \multirow{3}{*}{ Model } & \multicolumn{13}{|c|}{ Attack Confidence $=0.5$} \\
\hline & \multirow{2}{*}{ Attack Algorithm } & \multicolumn{3}{|c|}{$\mathrm{k}=$ top 2} & \multicolumn{3}{|c|}{$\mathrm{k}=$ top 4} & \multicolumn{3}{|c|}{$\mathrm{k}=$ top 6} & \multicolumn{3}{|c|}{$\mathrm{k}=$ top 10} \\
\hline & & ANC $\downarrow$ & $\mathrm{AI} \downarrow$ & SR $\uparrow$ & ANC $\downarrow$ & AI $\downarrow$ & $\mathrm{SR} \uparrow$ & ANC $\downarrow$ & $\mathrm{AI} \downarrow$ & $\mathrm{SR} \uparrow$ & ANC $\downarrow$ & AI $\downarrow$ & SR $\uparrow$ \\
\hline \multirow{5}{*}{ LSTM } & SGS & 2.93 & 3.87 & 0.89 & 2.41 & 3.03 & 0.91 & 2.12 & 2.55 & 0.90 & 1.72 & 1.96 & 0.89 \\
\hline & FSGS & \multicolumn{12}{|c|}{$\mathrm{ANC}=1.00 \quad \mathrm{AI}=1.00 \quad \mathrm{SR}=1.00$} \\
\hline & GradAttack & 1.51 & 1.00 & 1.00 & 3.23 & 1.00 & 1.00 & 3.74 & 1.00 & 1.00 & 3.86 & 1.00 & 1.00 \\
\hline & OMPGS-Rand & 2.75 & 3.03 & 0.98 & 2.98 & 3.42 & 0.98 & 3.12 & 3.66 & 0.96 & 3.37 & 4.15 & 0.96 \\
\hline & OMPGS & 2.12 & 2.15 & 0.98 & 1.71 & 1.75 & 0.98 & 1.60 & 1.65 & 0.98 & 1.52 & 1.57 & 0.98 \\
\hline \multirow{5}{*}{ LSTM-Noise } & SGS & 3.39 & 4.45 & 0.90 & 2.76 & 3.39 & 0.86 & 2.47 & 2.98 & 0.89 & 1.98 & 2.25 & 0.89 \\
\hline & FSGS & \multicolumn{12}{|c|}{$\mathrm{ANC}=1.20 \quad \mathrm{AI}=1.22 \quad \mathrm{SR}=1.00$} \\
\hline & GradAttack & 1.59 & 1.00 & 1.00 & 2.43 & 1.00 & 1.00 & 3.62 & 1.00 & 1.00 & 5.97 & 1.0 & 1.00 \\
\hline & OMPGS-Rand & 2.33 & 2.56 & 0.99 & 2.70 & 3.19 & 0.99 & 2.93 & 3.55 & 1.0 & 3.23 & 4.12 & 0.99 \\
\hline & OMPGS & 1.78 & 1.82 & 0.99 & 1.57 & 1.61 & 1.00 & 1.47 & 1.49 & 1.00 & 1.40 & 1.41 & 1.00 \\
\hline \multirow{5}{*}{ LSTM-Sub } & SGS & 2.96 & 3.96 & 0.96 & 2.47 & $\begin{array}{c}.03 \\
\end{array}$ & 0.93 & 2.18 & 2.51 & 0.94 & $\overline{1.78}$ & 1.93 & 0.94 \\
\hline & FSGS & \multicolumn{12}{|c|}{$\mathrm{ANC}=1.00 \quad \mathrm{AI}=1.00 \quad \mathrm{SR}=1.00$} \\
\hline & GradAttack & 1.20 & 1.00 & 1.00 & 1.34 & 1.00 & 1.00 & 1.34 & 1.00 & 1.00 & 1.40 & 1.00 & 1.00 \\
\hline & OMPGS-Rand & 1.69 & 1.78 & 0.99 & 2.29 & 2.62 & 0.99 & 2.59 & 3.12 & 0.99 & 3.02 & 3.84 & 0.99 \\
\hline & OMPGS & 1.01 & 1.01 & 0.99 & 1.01 & 1.01 & 0.99 & 1.01 & 1.01 & 0.99 & 1.01 & 1.01 & 0.99 \\
\hline \multicolumn{14}{|c|}{ Attack Confidence $=0.7$} \\
\hline \multirow{5}{*}{ LSTM } & SGS & 3.03 & 4.03 & 0.91 & 2.52 & 3.15 & 0.91 & 2.11 & 2.56 & 0.90 & 1.67 & 1.91 & 0.90 \\
\hline & FSGS & \multicolumn{12}{|c|}{$\mathrm{ANC}=1.00 \quad \mathrm{AI}=1.00 \quad \mathrm{SR}=1.00$} \\
\hline & GradAttack & 1.50 & 1.00 & 0.99 & 3.23 & 1.00 & 0.99 & 3.76 & 1.00 & 0.99 & 3.88 & 1.00 & 0.99 \\
\hline & OMPGS-Rand & 2.78 & 3.08 & 0.99 & 3.11 & 3.61 & 0.99 & 3.23 & 3.87 & 0.97 & 3.41 & 4.26 & 0.91 \\
\hline & OMPGS & 2.18 & 2.22 & 0.98 & 1.75 & 1.79 & 0.98 & 1.69 & 1.75 & 0.98 & 1.56 & 1.64 & 0.96 \\
\hline \multirow{5}{*}{ LSTM-Noise } & SGS & 3.34 & 4.38 & 0.87 & 2.79 & 3.56 & 0.90 & 2.45 & 2.97 & 0.89 & 2.45 & 2.97 & 0.89 \\
\hline & FSGS & \multicolumn{7}{|c|}{ ANC $=1.00$} & \multicolumn{5}{|c|}{$\mathrm{SR}=1.00$} \\
\hline & GradAttack & 1.59 & 1.00 & 0.99 & 2.44 & 1.00 & 0.99 & 3.58 & 1.00 & 0.99 & 5.96 & 1.00 & 0.99 \\
\hline & OMPGS-Rand & 2.45 & 2.71 & 0.99 & 2.83 & 3.40 & 0.99 & 3.06 & 3.72 & 1.00 & 3.24 & 4.02 & 0.91 \\
\hline & OMPGS & 1.95 & 2.02 & 0.99 & 1.70 & 1.75 & 1.00 & 1.63 & 1.65 & 1.00 & 1.58 & 1.59 & 0.99 \\
\hline \multirow{5}{*}{ LSTM-Sub } & SGS & 3.07 & 4.16 & 0.95 & 2.56 & 3.20 & 0.97 & 2.29 & 2.73 & 0.96 & 1.96 & 2.17 & 0.95 \\
\hline & FSGS & \multicolumn{12}{|c|}{$\mathrm{ANC}=1.00 \quad \mathrm{AI}=1.00$} \\
\hline & GradAttack & 1.22 & 1.00 & 0.98 & 1.36 & 1.00 & 0.97 & 1.35 & 1.00 & 0.97 & 1.45 & 1.00 & 0.97 \\
\hline & OMPGS-Rand & 1.70 & 1.80 & 1.00 & 2.27 & 2.64 & 1.00 & 2.65 & 3.20 & 1.00 & 3.05 & 3.92 & 1.00 \\
\hline & OMPGS & 1.04 & 1.04 & 1.00 & 1.02 & 1.02 & 1.00 & 1.01 & 1.01 & 1.00 & 1.02 & 1.02 & 1.00 \\
\hline
\end{tabular}

Electronic Health Records (EHR) [15]. The real-world EHR dataset consists of time-ordered medical visit records of 7314 patients. One patient data instance is organized as a tensor $\mathbf{x} \in$ $R^{200 * 4130 * 70}$, with 200 medical visits, 4130 diagnosis codes ${ }^{1}$, each of which is embedded as a 70-dim vector. A classifier is built for a binary task: predicting the risk of a patient suffering a heart disease. The evasion attack is simply to flip the binary classification output by changing the presence or absence of a code in one visit.

\subsection{Experimental Setup}

We instantiate the attackability study to the standard LSTM. To demonstrate the link between the regularity of the classifier and its attackability, we train three LSTM classifiers following different regularity constraints for each dataset: 1) LSTM: standard LSTM without any additional constraints, whose attack objective is weakly submodular with low submodularity ratio; 2) LSTM-Sub: LSTM with positiveness constraints proposed in [29], whose attack objective is strictly submodular according to Corollary 2.2; 3) LSTM-Noise: LSTM with parameter truncation, i.e., any parameters with their values less than -1 are truncated and assigned randomized positive values. To show how the attack methods perform given different levels of attack difficulty, we require each attack method to cause misclassification with a classification probability of 0.5 and 0.7 , noted as Attack Confidence Threshold. We include 4 state-of-theart greedy-search based attack methods, as well as the proposed

\footnotetext{
${ }^{1}$ For the patients with less than 200 visits, we pad the empty observations by setting the corresponding $b_{i}^{j}=0$. One code is the occurrence of a disease, a symptom, or an abnormal finding, see http://www.icd9data.com/.
}

OMPGS in the study.

1. Stochastic Greedy Search (SGS) [19]: SGS selects randomly a subset of attributes as the candidate of the greedy search in each iteration. Compared to FSGS, SGS is more efficient since it doesn't traverse every unselected attribute. As a price to pay, the approximation ratio of SGS degrades.

2. Gradient-based Attack (GradAttack) [29]. It follows the same attack objective definition in Eq. (1) and also uses gradients to guide the attribute selection. However, it only considers the new attributes contributing the largest marginal gain. According to Eq.1, it only searches a subset of the potentially feasible candidates, which inevitably cause loss of approximation quality.

3. OMPGS-Rand, a variant of OMPGS. It selects randomly one attribute from those with largest gradient magnitude in each iteration. It borrows the random sampling spirit from $S G S$ to reduce the computational cost. It is evaluated to demonstrate the necessity of combining the gradient's guidance and the greedy search.

4. FSGS. It is presented by Algorithm 1.

Comparison of these methods to our proposed OMPGS targets on 1) showing the computational efficiency and attack performances of our proposed OMPGS; and 2) the attackability of an evasion attack task is independent from the choice of specific attack methods. It depends on the functional characteristics of the classifier and the characteristics of the data on which the classifier is applied. For $S G S$ and $O M P G S$, we vary the number of candidate attributes $\mathbf{k}$ in each iteration ( $\mathbf{k}=2,4,6,10$ in Table.1 and Table.2). A higher $\mathbf{k}$ indicates a larger exploration range of greedy search, thus usually brings better attack performances. In contrast, GradAttack prefers smaller k. In 
Table 2: Attack Performances on EHR dataset

\begin{tabular}{|c|c|c|c|c|c|c|c|c|c|c|c|c|c|}
\hline \multirow{3}{*}{ Model } & \multicolumn{13}{|c|}{ Attack Confidence $=0.5$} \\
\hline & \multirow{2}{*}{ Attack Algorithm } & \multicolumn{3}{|c|}{$\mathrm{k}=$ top 2} & \multicolumn{3}{|c|}{$\mathrm{k}=$ top 4} & \multicolumn{3}{|c|}{$\mathrm{k}=\operatorname{top} 6$} & \multicolumn{3}{|c|}{$\mathrm{k}=$ top 10} \\
\hline & & ANC $\downarrow$ & AI $\downarrow$ & $\mathrm{SR} \uparrow$ & ANC $\downarrow$ & AI $\downarrow$ & $\mathrm{SR} \uparrow$ & $\overline{A N C} \downarrow$ & $\mathrm{AI} \downarrow$ & $\mathrm{SR} \uparrow$ & ANC $\downarrow$ & AI $\downarrow$ & $\mathrm{SR} \uparrow$ \\
\hline \multirow{5}{*}{ LSTM } & SGS & 3.65 & 5.13 & 0.10 & 3.35 & 4.72 & 0.14 & 2.98 & 4.07 & 0.12 & 3.63 & 5.19 & 0.25 \\
\hline & FSGS & \multicolumn{12}{|c|}{$\begin{array}{lll}\mathrm{ANC}=1.00 & \mathrm{AI}=1.00 & \mathrm{SR}=1.00\end{array}$} \\
\hline & GradAttack & 3.06 & 1.84 & 0.98 & 4.26 & 1.27 & 0.99 & 5.30 & 1.06 & 0.98 & 6.35 & 1.00 & 0.98 \\
\hline & OMPGS-Rand & 2.38 & 2.45 & 0.97 & 2.58 & 2.73 & 0.97 & 2.85 & 3.06 & 0.97 & 2.91 & 3.25 & 0.97 \\
\hline & OMPGS & 2.06 & 2.08 & 0.98 & 1.92 & 1.94 & 0.98 & 1.89 & 1.91 & 0.98 & 1.82 & 1.83 & 0.98 \\
\hline \multirow{5}{*}{ LSTM-Noise } & SGS & 4.43 & 6.5 & 0.06 & 3.79 & 5.59 & 0.09 & 3.28 & 4.68 & 0.08 & 2.78 & 3.48 & 0.07 \\
\hline & FSGS & \multicolumn{12}{|c|}{$\mathrm{ANC}=1.00 \quad \mathrm{AI}=1.00$} \\
\hline & GradAttack & 3.74 & 2.74 & 0.97 & 5.03 & 1.40 & 0.97 & 5.81 & 1.08 & 0.97 & 8.4 & 1.0 & 0.97 \\
\hline & OMPGS-Rand & 3.21 & 3.30 & 0.97 & 3.47 & 3.67 & 0.97 & 3.67 & 3.90 & 0.97 & 3.90 & 4.24 & 0.96 \\
\hline & OMPGS & 2.97 & 3.00 & 0.98 & 2.96 & 3.0 & 0.98 & 3.01 & 3.05 & 0.98 & 3.06 & 3.12 & 0.98 \\
\hline \multirow{5}{*}{ LSTM-Sub } & SGS & 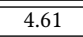 & 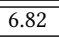 & 0.45 & 4.25 & 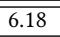 & 0.48 & 3.66 & 0.48 & 0.44 & 3.46 & 4.78 & 0.45 \\
\hline & FSGS & \multicolumn{12}{|c|}{$\begin{array}{ll}\mathrm{ANC}=1.00 & \mathrm{AI}=1.00\end{array}$} \\
\hline & GradAttack & 1.96 & 1.79 & 0.99 & 3.48 & 1.11 & 0.99 & 5.01 & 1.03 & 0.99 & 7.67 & 1.00 & 0.99 \\
\hline & OMPGS-Rand & 1.91 & 1.98 & 0.98 & 2.21 & 2.33 & 0.98 & 2.32 & 2.52 & 0.98 & 2.52 & 2.83 & 0.98 \\
\hline & OMPGS & 1.64 & 1.67 & 0.99 & 1.44 & 1.46 & 0.99 & 1.47 & 1.50 & 0.99 & 1.47 & 1.48 & 0.99 \\
\hline \multicolumn{14}{|c|}{ Attack Confidence $=0.7$} \\
\hline \multirow{5}{*}{ LSTM } & SGS & 4.56 & 7.32 & 0.15 & 4.36 & 6.46 & 0.16 & 4.14 & 6.02 & 0.16 & 4.18 & 6.37 & 0.28 \\
\hline & FSGS & \multicolumn{12}{|c|}{$\mathrm{ANC}=1.00 \quad \mathrm{AI}=1.00$} \\
\hline & GradAttack & 3.27 & 2.01 & 0.98 & 4.33 & 1.28 & 0.99 & 5.44 & 1.15 & 0.99 & 6.28 & 1.0 & 0.97 \\
\hline & OMPGS-Rand & 2.55 & 2.64 & 0.97 & 2.81 & 2.98 & 0.96 & 2.98 & 3.23 & 0.97 & 3.25 & 3.58 & 0.97 \\
\hline & OMPGS & 2.31 & 2.32 & 0.98 & 2.05 & 2.06 & 0.98 & 2.02 & 2.05 & 0.98 & 2.02 & 2.05 & 0.98 \\
\hline \multirow{5}{*}{ LSTM-Noise } & SGS & 5.16 & 7.58 & 0.10 & 4.35 & 6.45 & 0.08 & 4.30 & 6.69 & 0.10 & 4.09 & 6.23 & 0.17 \\
\hline & FSGS & \multicolumn{7}{|c|}{$\mathrm{ANC}=1.00 \quad \mathrm{AI}=1.20$} & \multicolumn{5}{|c|}{$\mathrm{SR}=1.00$} \\
\hline & GradAttack & 3.86 & 2.74 & 0.97 & 5.35 & 1.53 & 0.97 & 6.03 & 1.10 & 0.97 & 8.57 & 1.00 & 0.96 \\
\hline & OMPGS-Rand & 3.36 & 3.47 & 0.97 & 3.88 & 4.09 & 0.97 & 3.90 & 4.16 & 0.97 & 4.22 & 4.54 & 0.94 \\
\hline & OMPGS & 3.15 & 3.20 & 0.97 & 3.16 & 3.21 & 0.97 & 3.18 & 3.24 & 0.98 & 3.23 & 3.33 & 0.98 \\
\hline \multirow{5}{*}{ LSTM-Sub } & SGS & 5.19 & 8.00 & 0.55 & 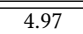 & 7.62 & 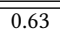 & 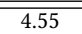 & $\overline{26.83}$ & 0.54 & 4.13 & 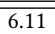 & 0.54 \\
\hline & FSGS & \multicolumn{7}{|c|}{$\overline{\mathrm{ANC}}=1.03 \quad \mathrm{AI}=1.04$} & \multicolumn{5}{|c|}{$\overline{\mathrm{SR}}=1.00$} \\
\hline & GradAttack & 1.94 & 1.55 & 0.98 & 3.36 & 1.12 & 0.99 & 5.02 & 1.01 & 0.98 & 7.63 & 1.00 & 0.99 \\
\hline & OMPGS-Rand & 2.03 & 2.08 & 0.98 & 2.31 & 2.47 & 0.98 & 2.48 & 2.73 & 0.98 & 2.63 & 2.99 & 0.98 \\
\hline & OMPGS & 1.78 & 1.82 & 0.99 & 1.60 & 1.63 & 0.99 & 1.55 & 1.56 & 0.99 & 1.56 & 1.57 & 0.99 \\
\hline
\end{tabular}

Table 3: Usability of the classifier on IPS dataset

\begin{tabular}{|c|c|c|c|}
\hline Classifier & Accuracy & Macro F1 & AUC \\
\hline LSTM & 0.9597 & 0.9432 & 0.9872 \\
\hline LSTM-Noise & 0.9668 & 0.9533 & 0.9870 \\
\hline LSTM-Sub & 0.8867 & 0.8687 & 0.9204 \\
\hline
\end{tabular}

Table 4: Usability of the classifier on EHR dataset

\begin{tabular}{|c|c|c|c|}
\hline Classifier & Accuracy & F1 & AUC \\
\hline LSTM & 0.9321 & 0.8831 & 0.9096 \\
\hline LSTM-Noise & 0.9277 & 0.8710 & 0.8948 \\
\hline LSTM-Sub & 0.9295 & 0.8730 & 0.8944 \\
\hline
\end{tabular}

each iteration, GradAttack selects k candidates of greatest gradient magnitudes. It is designed to traverse all possible combinations of the $\mathbf{k}$ attributes and take the best combination. A larger $\mathbf{k}$ usually introduces unnecessary attribute changes or changes with adverse effects on attack. The benchmark metrics used in the study are explained in details in the appendix.

\subsection{Results on IPS and EHR Datasets}

Table 1 and Table 2 illustrate the performances of all the attack methods on the 3 LSTM models. Table 3 and Table 4 show the classification accuracy of different LSTM-based classifiers on both datasets. We can summarize the observations as follows:

First, the utility scores indicate the usability of the real-world classification tasks. It is not surprising to see that LSTM enjoys the highest accuracy on both datasets. In contrast, LSTM-Sub, due to the positiveness constraint, performs relatively poorly. As expected, truncation noise doesn't impact much the classification of LSTMNoise thanks to the highly redundant network architecture.
Second, LSTM-sub is easier to attack (or more "attackable") than LSTM and LSTM-Noise, because it needs the least number of attribute changes and objective function queries. This observation confirms what we reveal earlier: for evasion attack on discrete data, a better regularized and bounded function form of the targeted classifier can improve the attackability of the evasion attack task. In contrast, attack against LSTM-Noise is slightly easier than LSTM on IPS data set but significantly more difficult on EHR data set. The explanation behind the inconsistency is as follows. The classifier becomes generally less smooth due to the truncation noise, which hence makes the corresponding attack objective further from strict submodularity. As a result, the attack performances against LSTMNoise become less tightly bounded by the regularity of the classifier according to Theorem 1, while more data-dependent.

Third, FSGS runs slowly on both datasets, while presents the best attack performances. This observation is highly consistent with the quality bound of $F S G S$ given by Theorem 2. FSGS can provide the superior approximation quality over any greedy-search based variant, no matter whether the attack objective is submodular or weakly-submodular. Both SGS and GradAttack improve computational complexity by reducing the number of candidate attributes in each iteration. Nevertheless, they don't consider explicitly how to minimize the loss of the quality of the solution.

Last, our proposed $O M P G S$ method always has high success rates and finds quickly the valid attacks (1-2 changes to make in one instance), because it combines the merit of orthogonal matching pursuit (OMP) and greedy search. On one hand, OMP helps to narrow down the greedy search to the attributes that are the 
most likely to be useful in the attack. On the other hand, OMPGS still conducts greedy search to explore the feasible subset of attributes improving the attack objective. OMPGS provides a good trade-off between exploration and exploitation in this sense. Without greedy search, the evasion attack performance of OMPGS-Rand is significantly worse than that of OMPGS.

\subsection{Sensitivity of the Selected Attributes}

For each data set, we conduct one-factor-at-a-time sensitivity analysis [6] over the discrete attributes. Given a data instance, we change each attribute while keeping all the others fixed. The averaged change of the probabilistic classification output over all the testing instances is used as the attribute-wise sensitivity measurement. A larger average change indicates that the classifier's output is more sensitive to the change over the corresponding attribute. Following Eq.9, we launch OMPGS based attack against LSTM-Sub on both datasets. The attack spins till Attack Confidence $=0.5$.

For attacking LSTM-Sub, we finally select 5 attributes on IPS data and 7 attributes on EHR data. On IPS data set (1103 attributes), 3 of the $\mathbf{5}$ attributes also appear as the top $50 \%$ sensitive attributes. Especially, 2 of them are ranked as the top $12 \%$ sensitive attributes. On EHR data set (4130 attributes), 5 of the $\mathbf{7}$ attributes show up in the list of the top 50\% sensitive attributes. Furthermore, 3 of them are ranked as top $12 \%$ sensitive attributes. The interesting overlapping between the attributes useful for attack and the topranked sensitive attributes confirms our intuition about stability of the classification model. Though highly sensitive attributes can help better capture the difference between different classes, they can also increase adversarial vulnerability of the classifier [35]. Moreover, the observation unveils that evasion attack can also be used to exploit the statistical characteristics (e.g., attribute sensitivity) of the training data of the classification system. It can steal privacysensitive information of the training data.

\section{CONCLUSION}

In this paper, we explore attackability guarantees of evasion attack against a targeted classifier on discrete input. We unveil the regularity constraints over the function form of the targeted classifier Despite of the general NP-hard complexity, evasion attack targeted at a classifier bounded by the constraints enjoys the approximately diminishing return property of weak submodularity. It can thus deliver an efficient attack via fast greedy search and provide provable attack performances. Furthermore, following the framework of the attackability study, we propose an orthogonal matching pursuit guided greedy search to achieve a good balance between economic candidate attribute search and efficient attack. Both theoretical and empirical study confirm the merits of the proposed method. For a classifier with discrete inputs, our work reveals theoretically a link between functional characteristics of the classifier and its adversarial vulnerability. Our study is thus useful for improving robustness of the classifier facing hostile adversarial threats.

\section{ACKNOWLEDGMENTS}

Our research in this publication was supported by funding from King Abdullah University of Science and Technology (KAUST), under award number FCC/1/1976-19-01 and KAUST AI Initiative, and NSFC No. 61828302.

\section{REFERENCES}

[1] A. Akbarnejad and S. Günnemann. Adversarial attacks on node embedding via graph poisoning. In ICML, 2019.

[2] I.M. Alabdulmohsin., X. Gao, and X. Zhang. Adding robustness to support vector machines against adversarial reverse engineering. In CIKM, page 231-240, 2014.

[3] I.M. Alabdulmohsin., X. Gao, and X. Zhang. Efficient active learning of halfspaces via query synthesis. In $A A A I$, page 2483-2489, 2015.

[4] B. Nelson B. Biggio and P. Laskov. Poisoning attacks against support vector machines. In ICML, 2012.

[5] J. Bilmes and W. Bai. Deep submodular functions. arXiv preprint arXiv:1701.08939, 2017.

[6] Chai T M. M Tang Y B. DR B. NJ V. SA C. GJ B. I B.JA M. SA S. C S. JL C. JE, C. GR and S. CO. Photosynthetic control of atmospheric carbonyl sulfide during the growing season. Science, 322, 2008.

[7] Nicholas Carlini and David Wagner. Towards evaluating the robustness of neural networks. In IEEE S\&P, 2017.

[8] Nicholas Carlini and David Wagner. Audio adversarial examples: Targeted attacks on speech-to-text. In SPW, 2018.

[9] L. Chen, M. Feldman, and A. Karbasi. Weakly submodular maximization beyond cardinality constraints: Does randomization help greedy? In ICML, 2017.

[10] A. Akbarnejad D. Zügner and S. Günnemann. Adversarial attacks on neural networks for graph data. In $K D D, 2018$.

[11] A. Das and D. Kempe. Submodular meets spectral: Greedy algorithms for subset selection, sparse approximation and dictionary selection. In ICML, 2011.

[12] D.Zugner, A.Akbarnejad, and S.Gunnemann. Adversarial attacks on neural networks for graph data. In IFCAI, 2019.

[13] A. G. Dimakis E. R. Elenberg, R. Khanna and Sahand Negahban. Restricted strong convexity implies weak submodularity. Annuals of Statistics, 2016.

[14] Javid Ebrahimi, Anyi Rao, Daniel Lowd, and Dejing Dou. HotFlip: White-box adversarial examples for text classification. In ACL, 2018.

[15] Q. Suo Q. You J. Zhou F. Ma, J. Gao and A. Zhang. Risk prediction on electronic health records with prior medical knowledge. In $K D D, 2018$.

[16] J. Gao, J. Lanchantin, M. L. Soffa, and Y. Qi. Black-box generation of adversarial text sequences to evade deep learning classifiers. In $S P W$, pages 50-56, 2018.

[17] Z. Gong, W. Wang, B. Li, D. Song, and W. Ku. Adversarial texts with gradient methods. ArXiv, abs/1801.07175, 2018.

[18] I. Goodfellow, J. Shlens, and C. Szegedy. Explaining and harnessing adversarial examples. In ICLR, 2015.

[19] A. Hassidim and Y. Singer. Robust guarantees of stochastic greedy algorithms. In ICLR, 2017.

[20] K. He, X. Zhang, S. Ren, and J. Sun. Deep residual learning for image recognition. In CVPR, 2016.

[21] V. Kuleshov, S. Thakoor, T. Lau, and S. Ermon. Adversarial examples for natural language classification problems. In ICLR, 2018.

[22] T. Miyato, A. M. Dai, and I.J. Goodfellow. Adversarial training methods for semi-supervised text classification. In ICLR, 2016.

[23] G. Nemhauser M.L. Fisher and L.A. Wolsey. An analysis of approximations for maximizing submodular set functions. Mathematical Programming, 8, 1978.

[24] G. Nemhauser and L.A. Wolsey. An anlaysis of approximations for maximizing submodular set functions. Mathematical Programming, 14, 1978.

[25] G. Nemhauser and L.A. Wolsey. Best algorithms for approximating the maximum of a submodular set function. Mathematics of Operations Research, 3, 1978.

[26] Christos H. Papadimitriou and Kenneth Steiglitz. Combinatorial Optimization: Algorithms and Complexity. Prentice-Hall, Inc., 1982.

[27] N. Papernot, P. D. McDaniel, A. Swami, and R. E. Harang. Crafting adversarial input sequences for recurrent neural networks. In MILCOM, 2016.

[28] Y. C. Pati, R. Rezaiifar, and P. S. Krishnaprasad. Orthogonal matching pursuit: recursive function approximation with applications to wavelet decomposition. In ACSSC, 1993.

[29] L. Qi, L. Wu, P. Chen, A. Dimakis, and and M. Witbrock I. Dhillon. Discrete attacks and submodular optimization with applications to text classification. In SysML, 2019.

[30] S. Samanta and S. Mehta. Towards crafting text adversarial samples. CoRR, abs/1707.02812, 2017.

[31] C. Szegedy, W. Zaremba, I. Sutskever, J. Bruna, D. Erhan, I. J. Goodfellow, and R. Fergus. Intriguing properties of neural networks. In ICLR, 2013.

[32] H. Xu, Y. Ma, H. Liu, D. Deb, H. Liu, J. Tang, and A. K. Jain. Adversarial attacks and defenses in images, graphs and text: A review, 2019.

[33] P. Yang, J. Chen, C. Hsieh, J. Wang, and M. I. Jordan. Greedy attack and gumbel attack: Generating adversarial examples for discrete data. ArXiv, abs/1805.12316, 2018.

[34] Y.Yao, B.Viswanath, J.Cryan, H.Zheng, and B.Zhao. Automated crowdturfing attacks and defenses in online review systems. In ACM CCS, 2017.

[35] F. Zhang, P. P. K. Chan, B. Biggio, D. S. Yeung, and F. Roli. Adversarial feature selection against evasion attacks. IEEE Transactions on Cybernetics, 46, 2016. 


\section{A DATASET INFORMATION}

We include two datasets collected from real-world classification applications on cyber security and electronic medical service, as summarized as follows:

Intrusion Prevention System Dataset (IPS). Modern cyberattacks have reached high levels of complexity. An attacker who is trying to compromise a computer system has to perform a series of attack steps (i.e., reconnaissance, exploitation, and persistence) to achieve such goal. For each of the attack steps, attackers have a choice of executing a series of malicious actions, such as exploiting Apache Struts or exploiting Wordpress file download, which are usually scripted and automated. We collect one day of IPS records from 242,467 endpoint devices containing 29,641 time series of attack events. Each sequence instance is composed by 20 attack steps. On each attack step, the attacker can choose one of 1103 different malicious actions registered as highly threatening ones. Thus one data instance of IPS data is given as $\mathbf{x} \in R^{20 * 1103 * 70}$ according to the definition given in Section.3. Each of the 1103 malicious actions is projected to a 70-dimensional embedding vector. In our study, each sequence instance is used as input to the classification system, which predicts the most likely attack operation conducted at the immediately successive step of the sequence. Based on the prediction output, security analysts can proactively take prevention actions. We focus on predicting the occurrence of the two most threatening actions related to recently uncovered vulnerability. We thus study a 3-class classification task: the 2 highly malicious actions and all the others as the third class.

Electronic Health Records (EHR) [15]. The real-world EHR dataset consists of time-ordered medical visit records of 7314 patients. Each patient has from 4 to 200 medical visits. Each visit record is composed by a subset of 4130 discrete ICD9 diagnosis $\operatorname{codes}^{2}$. Each diagnosis code represents occurrence of a disease, a symptom, or an abnormal finding. Using the historical EHR data of patients, we can predict the risk of patients suffering the target diseases. In this experiment, our target is a binary classification task: we forecast whether a patient will suffer heart failure disease in the future. In our experiments, a data instance of EHR data set is organized as a tensor $\mathbf{x} \in R^{200 * 4130 * 70}$ with each of the 4130 diagnosis codes projected to a 70-dimensional embedding vector. For the patients with less than 200 visits, we pad the empty observations by setting the corresponding $b_{i}^{j}=0$.

We split randomly each dataset into 3 non-overlapped subsets for training, testing and evaluating the attack performances. For IPS data, 21,214 and 7,427 sequence instances are used to train and evaluate the classification accuracy of the classifier. The left 1,000 instances are used for benchmarking the performances of the evasion attack. On IPS data, we focus on the adversarial attribute change by replacing the original action at a given attack step with a new action. For EHR data, we use 5,679 and 1,135 patient visit instances to train and test the classifier. The rest 500 visit instances are used to attack. As the diagnosis code in each visit instance is binary, we conduct the attack by simply flipping the codes.

\section{B EXPERIMENTAL SETUP}

We instantiate the attackability study to a popularly adopted RNN based classifier, standard Long Short-Term Memory (LSTM) model in the experiments. Without loss of generality, we use Tanh activation function in the LSTM classifier and exclude the dropout module. Theorem.1 applies to LSTM similarly as the simpler RNN architecture. To demonstrate the link between the regularity of the classifer and its attackability, we train three LSTM classifiers following different regularity constraints for each dataset. First, we use the standard LSTM mode without any additional constraints over the models' parameter. According to Corollary 2.1, the evasion attack objective targeted at the classifier is weakly submodular with low submodularity ratio. This classifier is referred as LSTM in the experiments. Similarly, we enforce the positiveness constraints on the classifier proposed in [29]. The resultant LSTM is strictly submodular according to Corollary 2.2. It is noted as LSTM-Sub in the followings. Though LSTM and LSTM-Sub share the similar level of smoothness, the activation function of LSTM-Sub is truncated to be positive and thus presents strongly concavity. In contrast, the activation function of LSTM is convex on the negative input and in general is not concave any more. As shown by Definition.1, the regularity parameter $m$ of $L S T M-S u b$ is large than that of LSTM. In other words, LSTM-Sub is more regularized than LSTM. We compare the attack performances against them to confirm our theoretical discussion and intuition: more regularized and bounded classifier is easier to be attacked with greedy-search based methods. Furthermore, we add additional parameter perturbations to LSTM by parameter truncation: any parameters with their values less than -1 are truncated and assigned randomized positive values. As per our empirical observation, most of LSTM's parameters are larger than -1 . Therefore, the parameter perturbation causes little changes of classification performances. But it can reduce the classifier's smoothness as the randomized parameter perturbation introduces unpredictable change to the first-order derivative of the classifier. We refer the noisy version of LSTM as LSTM-Noise. Comparing attack performances against LSTM and LSTM-Noise can demonstrate further that attackability of a less consistently regularized classifier is more difficult to be estimated.

The evasion attack task on IPS data is non-targeted. We aim at mis-classifying the attack sequence to any of the two classes other than the true class label. The evasion attack on EHR data simply flips the binary classification output. To show how the attack methods perform given different levels of attack difficulty, we require each attack method to cause misclassification with a classification probability of 0.5 and 0.7 respectively, as noted as Attack Confidence Threshold in the results. We include 4 state-of-the-art greedy-search based attack methods, as well as the proposed OMPGS in the study. The purpose is two-fold. Firstly we involve the baselines for comparative study, in order to show the computational efficiency and attack performances of our proposed OMPGS. Secondly, we show that the attackability of an evasion attack task is independent from the choice of specific attack methods. It depends on the functional characteristics of the classifier and the characteristics of the data on which the classifier is applied. Except FSGS, the other involved baseline approaches are as follows:

\footnotetext{
${ }^{2}$ http://www.icd9data.com/
} 
- Stochastic Greedy Search (SGS) [19]: SGS selects randomly a subset of attributes as the candidate of the greedy search in each iteration. Compared to FSGS, SGS is computationally more efficient since it doesn't traverse every unselected attribute. As a price to pay, the approximation ratio of SGS degrades, as shown in [19]. We believe that the proposed $O M P G S$ performs better. Benefited from the gradient information used in OMPGS the candidate set of the greedy search greatly shrinks while preserving attack effectivity.

- Gradient-based Attack (GradAttack) [29]. GradAttack follows the same attack objective definition in Eq.1 and also uses gradients to guide the attribute selection. However, it only considers the new attributes contributing largest marginal gain with respect to the currently best combination of attribute changes. According to Eq.1, GradAttack only searches a subset of the potentially feasible candidates in each iteration, which inevitably cause loss of approximation quality of the attack solution.

Besides, we also involve a variant of OMPGS, named as OMPGSRand. It works by selecting randomly one attributes from the attributes with largest gradient magnitude in each iteration. It is designed by borrowing the random sampling spirit from $S G S$ to reduce the computational cost. The purpose of involving OMPGSRand is to demonstrate the necessity of combining the gradient's guidance and the greedy search within the candidate attributes. For all the attack methods except FSGS, we vary the size of the candidate attribute set for greedy search in each iteration from 2 to 10 , noted as top2, top4, top6 and top10. We implement all the attack methods and the 3 LSTM based classifiers using the Python library PyTorch and conduct all the experiments on Linux server with 2 GPUs (GeForce 1080Ti) and 16-core CPU (Intel Xeon).
Benchmark Metric. We measure Accuracy Score, F1 score and AUC score to evaluate the usability of the trained LSTM-based classifiers. To illustrate the computational efficiency of different methods, we force all the methods except FSGS to halt after 60s and compare the success rate (noted as SR) of evasion attacks. A higher $S R$ value denotes a faster attack process. We allow FSGS to run for 3600 s and report the metrics after it stops. Furthermore, we record the average number of attribute $(A N C)$ and the average number of iteration $(A I)$ of each method to achieve the attack goal. Both metrics are used to measure attack efficiency. Note that $A N C$ does not necessarily equal to AI. According to Eq. (1), maximizing the set function based attack objective is not obliged to increase the support set $S$ in each iteration. A successful evasion attack with lower ANC indicates better preserving data integrity, thus more invisible to data sanitary check. ANC is thus the most important indicator measuring the effectiveness of attack methods. In contrast, $\mathbf{A I}$ is used to show the computational cost, as more iterations require more objective function evaluations.

We release the source codes for experimental study at https://github.com/X8GWRFJT/Attackability-Characterizationof-Adversarial-Evasion-Attack-on-Discrete-Data. 